\title{
'I Feel Fat': Weight-Related Stigma, Body Esteem, and BMI as Predictors of Perceived Competence in Physical Activity
}

\author{
Dorothy L. Schmalz \\ Department of Parks, Recreation, and Tourism Management, Clemson University, Clemson, SC, USA
}

\section{Key Words}

Stigma consciousness - Objective weight status .

Subjective weight status - Physical activity

\section{Summary}

Background: Efforts toward increasing participation in physical activity to prevent and treat overweight and obesity have as yet proved unsuccessful. Among the many barriers that discourage participation are weightrelated stigmas. The primary purpose of this study was to explore the relationship between consciousnesses of weight-related stigma and perceived competence in physical activity. A secondary purpose of the study was to explore the potential mediation effects of body esteem and body mass index (BMI) on the relationship. Consciousness of weight stigma, body esteem, and BMI are explored as predictors of perceived competence in physical activity. Methods: Participants included 76 adults who were enrolled in a weight management program. Data were collected using self-administered questionnaires. Path modeling was used to assess the relationships between stigma consciousness and perceived physical activity competence, and the potential mediation effects of BMI and body esteem. Results: Tests indicated that body esteem, but not BMI, mediated the relationship of consciousness of weight-related stigma and perceived physical activity competence. Conclusions: Consciousness of weight stigma, regardless of objective weight status, may negatively affect individuals' willingness to participate in physical activity. Findings such as these are important in furthering understanding the factors that affect behavior, and subsequently conducting programs to overcome perceptions of weight and physical activity to encourage participation.

\section{Introduction}

In the last decade, increasing rates of overweight and obesity and the importance of participation in physical activity have become issues of international concern. However, efforts toward increasing participation in physical activity have as yet proved unsuccessful. There are a number of constraints individuals face in regard to their participation in physical activity, such as lack of access and availability, lack of friends with whom to participate, and lack of familial support for participation [1]. In addition to environmental and social barriers such as those, participation in physical activity is complicated by psychological barriers such as acute sensitivity to perceived weight status and consciousness of weight stigma, lack of motivation, and low perceived competence [2-7]. Research has shown that the messages that people internalize as a result of concern of stigma to which they might be subjected affect them subliminally, and can greatly affect their approach to, and behavior in, different situations [8]. In the case of overweight and obesity, the degree to which a person is sensitive to weight-related stereotypes and subsequent stigma may proximally affect their perceived competence in physical activity, and distally affect their participation. Understanding the social-psychological mechanisms at work that serve as barriers to participation in physical activity and ways to overcome them are important steps that must be addressed in order to better program for and provide support to encourage participation.

\section{Background}

Weight-Related Stigma and Physical Activity

Stigmata are negative labels that are assigned to people or groups by society because of certain attributes they possess

Dr. Dorothy L. Schmalz

Department of Parks, Recreation, and Tourism Management Clemson University

263 Lehotsky Hall, Clemson, SC 29634, USA

Tel. +1 864 656-2184, Fax -2226

schmalz@clemson.edu 
that are considered different from the social norm [9]. In one of the earliest efforts toward defining and researching the phenomenon of social stigma, Goffman [10] made the observation that stigmata are a relationship between an attribute or characteristic, and the negative stereotypes associated with the characteristic. Link and Phelan [8] conceptualized stigma as a multi-level process in which a characteristic is associated with negative stereotypes, and individuals who possess the characteristic are subject to discrimination and reduced social status. Examples of attributes that are subject to stigma are race, religion, gender, and physical characteristics such as weight. As rates of overweight and obesity continue to climb [11], weight-related stigma has become well documented in the literature. Common weight-related stereotypes that fuel weight stigma are that overweight individuals are lazy, sloppy, unhappy, stupid, and that they lack motivation, willpower, education, and friends [12]. Experiences with, and internalization of, weight-related stereotypes has been shown to negatively affect individuals' psychological well-being [5, 13, 14], eating behaviors [14], and physical activity [2, 4-7]. Stereotypes that overweight people are 'lazy' and 'unmotivated' support personal and social misconceptions that physical activity is for thin people, thus perpetuating personally imposed barriers to participation among people who are sensitive to the stigmata assigned people based on weight [2, 4-7, 15]. Lacking the self-confidence or belief that one has the power and ability to participate in activities linked to weight management is likely to negatively affect perceived competence, and subsequently the pursuit and adoption of physical activity.

\section{Theoretical Framework}

The stigma consciousness construct was designed to assess idiosyncratic awareness and internalization of negative stereotypes that are assigned stigmatized individuals; in this case, weight stigma. Previous work incorporating the stigma consciousness construct has investigated people who are gay, lesbian, Black, and female [16, 17]. Results of previous studies suggest that individuals with higher stigma consciousness are more likely to curb behaviors if they fear participation would associate them with or expose them to stigma $[16,17]$. In this case, the stigma that overweight and obese people are lazy and inactive would negatively affect perceived competence and participation in physical activity among individuals who are highly stigma conscious. Although previous research has shown that being teased or the subject of weight stereotypes has negative effects on psychological well-being [18, 19], less research has explored the effects of personally possessing or internalizing weight stereotypes or stigma. Among other groups that are subject to stigma (race, gender), group members find solace with each other, and develop pride and unity in their similarity [20]. Individuals who are subject to weight stigmatization do not experience the same unification among group members, and often possess anti-fat biases themselves $[14,15,21,22]$. This is due in part to the perception that their group membership is temporary and controllable [19, 22, 23]. Consequently, overweight individuals do not benefit from the group mentality and support of similar others, which may further magnify the negative consequences of negative self-talk and internalization of weight stigma. Among the many attributes that are subject to stigma, weight-related stigma has been argued to be the most psychologically damaging to group members [19]. Unlike members of other stigmatized groups (e.g., race, sexual identity, gender), individuals who perceive themselves to be overweight internalize weight stigma and possess anti-fat biases themselves [23, 24]. Previous research has contended that this is due in part to the perceived controllability of the condition by in- and out-group members $[19,22,23]$. According to the stigma consciousness construct, people who are conscious of and internalize weightrelated stigmata are apt to disassociate from and even avoid participation in physical activity.

\section{'I Feel Fat': Body Mass Index and Body Esteem}

It is important to note that internalization of weight stereotypes and stigmata is not exclusive to overweight and obese people. Although weight is measurable objectively, most commonly by body mass index (BMI), people often cognitively miscalculate their weight status [25]. Individuals who may be overweight according to their BMI may cognitively consider themselves to be healthy. On the other hand, individuals who are at a healthy weight according to their BMI, may cognitively consider themselves to be overweight [25]. In a study exploring the relationship of BMI to a variety of perceived competence dimensions, Craft et al. [26] found that BMI was not a predictor of physical activity or athletic competence. The effects of weight stigma on participation in physical activity may be a mechanism of body esteem and the perception of 'feeling fat,' rather than actually being overweight by BMI standards. Previous research has found that sensitivity to weight stigma contributes to a reduction in effective coping, and increases negative self-talk and poor eating habits [27]. However, little has been done investigating the effects of weight stigma on participation in physical activity.

\section{Purpose of the Study}

The primary purpose of this study was to explore the relationship between consciousnesses of weight-related stigma and perceived competence in physical activity. A secondary purpose of the study was to explore the potential mediation effects of body esteem and BMI on the relationship. Four hypotheses guided the study: i) there will be a negative relationship between consciousness of weight-related stigma and perceived competence of physical activity, ii) body esteem will be positively related to perceived competence of physical activity, iii) BMI will not be related to perceived competence of physical activity, and iv) body esteem, but not BMI, will mediate the relationship between consciousness of weight-related stigma and perceived physical activity competence (fig. 1). This study 
Fig. 1. Hypothesized model of the effects of weight stigma, body mass index, and body esteem on perceived competence.

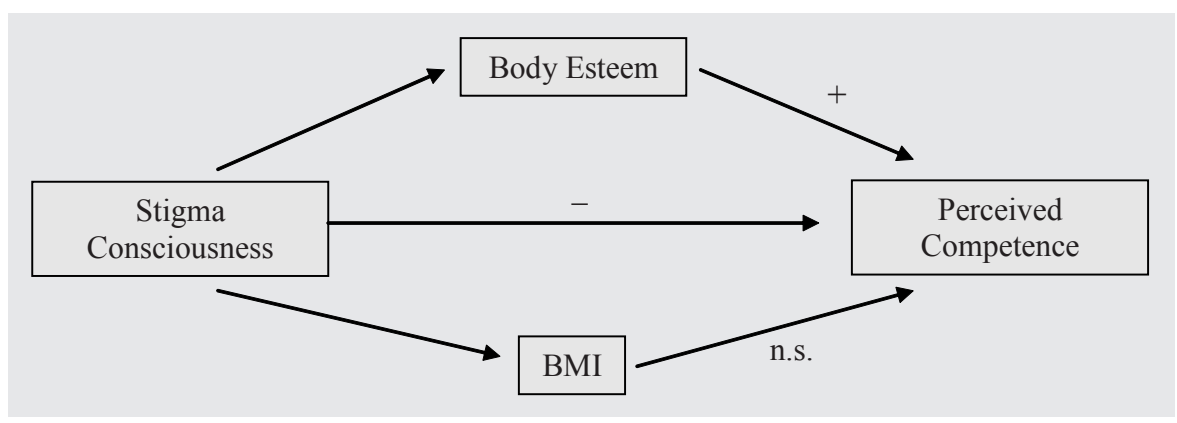

presents a distinct perspective of examining the role of perceived weight status in addition to objective weight on physical activity competence, in addition to an exploration of the effects of weight stigma on perceived competence.

\section{Participants and Methods}

\section{Participants}

Prior to data collection, human subjects approval was obtained from the Office of Research Protections at the university associated with the research. A snowball sampling method was used to recruit participants for the study. Initial participants were solicited at the front of a weight management center. The center served as a convenient location to identify individuals who have concerns about their weight, either objective or subjective. Individuals aged 18 years or older who were entering the center were asked to participate and whether they knew anyone else 18 years or older who would be willing to complete the survey. Interested participants were provided with self-administered questionnaires to take home and complete on their own time. Participants deposited their completed questionnaires in a locked box located at the center. The researcher and project assistants collected the contents of the box once each week, after all clients had left the center in order to maintain confidentiality and prevent any potential discomfort for study participants. Because participants were 18 years or older, and no identifying information was collected, participant consent was implied when they accepted and completed the questionnaire. Data were collected from 93 participants, 76 of whom completed the questionnaires used for this study in full and were used for data analysis. The majority of the sample was female $(n=83)$, white, non-Hispanic $(n=80)$, and 50 years or older $(n=54)$. Close to one third had some college or post-high school training $(\mathrm{n}=28)$, and one quarter had a college degree $(\mathrm{n}=20)$. Of the 90 participants who provided height and weight information, BMIs ranged from 19.5 to $48.0 ; 48 \%$ of the sample had a BMI of 30.0 or higher $(\mathrm{n}=45), 29 \%$ had a BMI between 25.0 and $29.9(\mathrm{n}=30)$, and $19 \%$ had a BMI within a healthy range below 24.9 $(\mathrm{n}=20 \%)$.

\section{Measures}

\section{Stigma Consciousness}

Stigma consciousness was assessed using the stigma consciousness questionnaire (SCQ) [16, 17], modified for weight stigma. The SCQ has been used to explore stigma consciousness among groups that are often subject to stigma based on sexual identity, gender, and race, but has not been used among overweight or obese populations. The SCQ was developed with the intention of being able to modify it to assess stigma consciousness among different groups. For example, an original, reverse-coded item in the scale ('stereotypes about women have not affected me person- ally') can be easily changed to address weight stigma ('stereotypes about weight have not affected me personally') for the purposes of the current study. The SCQ consists of 10 items to which participants are asked to respond on a 7-point scale ranging from 'strongly disagree' (1) to 'strongly agree' (7). In the final analyses, a high mean score on the SCQ meant that the participant had a high degree of stigma consciousness. Test-retest results of the 10-item SCQ in previous studies show that the scale has a satisfactory Chronbach's alpha of 0.72. During scale development, Pinel [16] predicted that the SCQ would be correlated with scales of related constructs such as self-consciousness and personal attributes. Correlation analyses confirmed discriminant and concurrent validity with the related scales.

\section{Perceived Competence in Physical Activity}

The physical activity dimension of the Harter Self-Perception Profile was used to measure perceived physical activity competence [28]. In its entirety, the Self-Perception Profile consists of 6 subscales: i) physical activity competence, ii) physical appearance competence, iii) social acceptance, iv) behavior competence, v) cognitive competence, and vi) global self-worth. Each dimension can be assessed independently of the others [28]. The physical activity dimension consists of 5 items that address the degree of competence an individual perceives in their participation in physical activity (e.g., 'I do well in all kinds of sports'). Participants are asked to respond on a 4-point scale ranging from 'really disagree' (1) to 'really agree' (4). Higher scores indicate higher perceived competence. Previous uses of the scale in studies have shown satisfactory Chronbach's alphas ranging from 0.80 to 0.88 . Previous studies have reported concurrent validity of the scale via correlations with related scales such as influences on physical activity and activity motivation.

\section{Body Esteem}

Body esteem was measured using the body esteem scale for adolescents and adults (BES) [29]. The BES consists of 22 items that explore degree of body image and confidence (e.g., 'I'm proud of my body'). Responses are coded on a 5-point scale ranging from 'never' (1) to 'always' (5). The scale consists of 3 subscales: i) appearance, ii) weight satisfaction, and iii) attribution (e.g., perceptions of other peoples' evaluation of one's body and appearance). Test-retest examinations have shown satisfactory reliabilities for the appearance, weight satisfaction, and attribution subscales, of $0.93,0.93$, and 0.75 respectively. All 3 dimensions of the BES were used to calculate an aggregate body esteem score in this study. The use of the aggregated scale has shown a test-retest reliability of 0.87 . In previous studies, the subscales of the body esteem scale have shown concurrent and predictive validity through correlations with related scales, such as weight concerns, BMI, and maladaptive eating behavior. The higher the mean on the BES, the higher the body esteem an individual has.

\section{$B M I$}

The instrument also included a demographic section in which participants were asked to self-report their height and weight in order to calculate BMI ((weight $(\mathrm{kg}) /$ height $\left.(\mathrm{m})^{2}\right)$. 


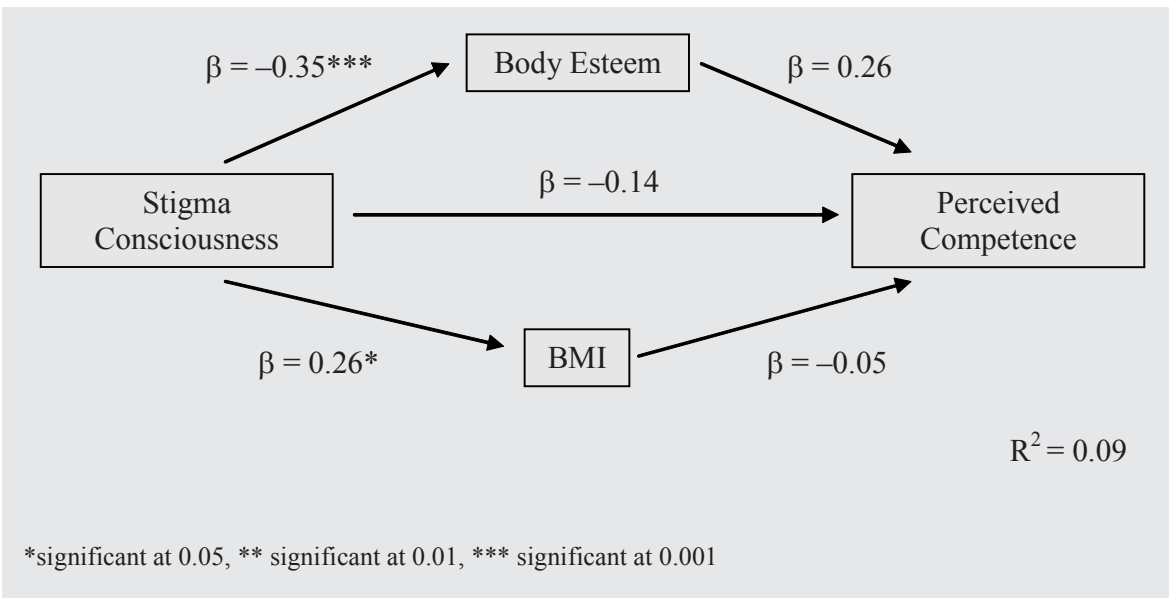

Fig. 2. Multiple mediation model of the effects of weight stigma, body mass index, and body esteem on perceived physical activity competence.

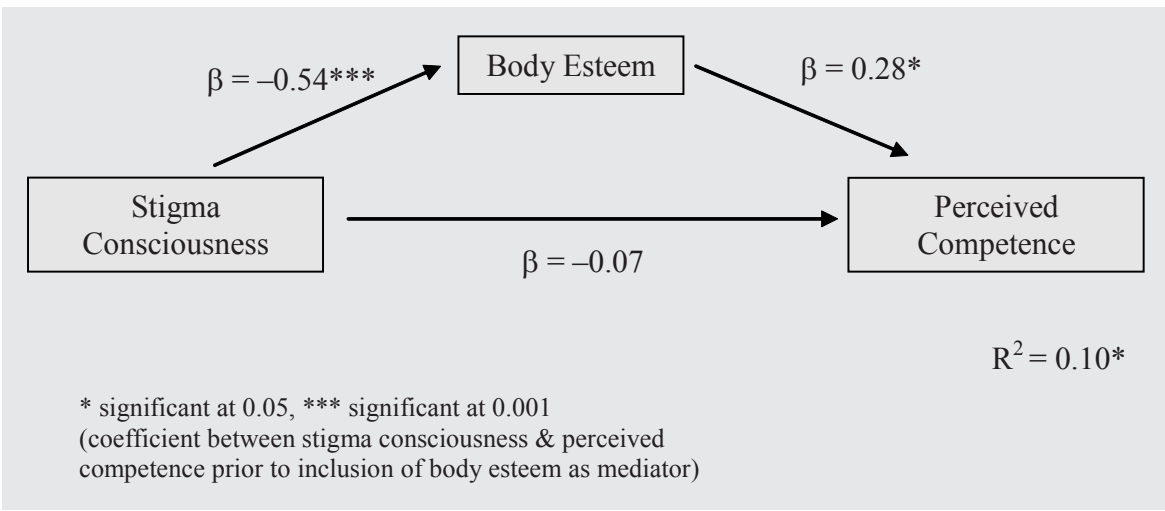

Fig. 3. Final mediation model of the effects of weight stigma, body mass index, and body esteem on perceived competence. independent variable has no effect on the dependent variable when the mediator is controlled, Baron and Kenny contend that perfect mediation is taking place. and subsequent emotional well-being has indicated little to no gender difference [25, 27]. Therefore, men and women were invited to participate in the study. A total of 93 adults completed the questionnaire, 17 of which did not complete all of the demographic information or scales used for this study (e.g., height and weight, SCQ, physical activity competence, and body esteem), so they were eliminated from analyses. Data from 76 participants were analyzed for this study. Data were analyzed using SPSS 16.0 (Chicago, IL, USA). Scale reliabilities were assessed using alpha coefficients [30]. Reliability estimates were 0.79 , 0.85 , and 0.91 for the stigma consciousness, perceived competence, and body esteem scales respectively. Prior to performing tests on the hypotheses, bivariate correlation analyses were run to test the relationships between the variables. Relationships between all 3 dependent variables and the independent variable were significant. The 4 hypotheses that guided the study were tested using linear regression path analyses. Standardized effects and $p$ values $\leq 0.05$ were used to determine significance. Rules for testing for mediation as outlined by Baron and Kenny [31] were followed. In a series of 3 regression equations, relationships between the independent (SCQ), mediator (BE), and dependent (perceived competence in physical activity) variables should be determined. The 3 regressions should be as follows: first, the effect of the independent variable on the mediator variable should be assessed; second, the effect of the independent variable on the dependent variable; and third, the effect of the mediator on the dependent variable. If, in the third regression when the mediator was controlled, the effect of the independent variable on the dependent variable is less than in the second, the mediation is taking place. In the event that the

\section{Results}

\section{Hypothesis Testing}

Four hypotheses guided the study: i) there will be a negative relationship between consciousness of weight-related stigma and perceived competence of physical activity, ii) body esteem will be positively related to perceived competence of physical activity, iii) BMI will not be related to perceived competence of physical activity, and iv) body esteem, but not BMI, will mediate the relationship between consciousness of weight-related stigma and perceived physical activity competence. The first, second, and third hypotheses required individual testing of the dependent variables on perceived physical activity competence. The first hypothesis was supported. As predicted, stigma consciousness was weakly and negatively related to perceived physical competence $(r=-0.22, p<0.05)$. The second hypothesis was also supported. Tests showed a strong, positive relationship between body esteem and perceived physical competence $(\mathrm{r}=0.31, \mathrm{p}<0.01)$. The third hypothesis posited that BMI would not be related to perceived 
physical competence. This hypothesis was supported; however, the correlation analysis indicated that BMI was negatively, though weakly, related to perceived physical competence $(\beta=-0.27, p<0.05)$. To test potential mediation as proposed in the fourth hypothesis, stigma consciousness, body esteem, and BMI were entered into a mediation model simultaneously to test their relationship to perceived physical activity competence. The model was not significant $\left(\mathrm{R}^{2}=0.09, \mathrm{p}>\right.$ $0.05)$, and BMI had the least effect $(\beta=-0.05, \mathrm{p}=0.70)$ in the path analysis (fig. 2). Because BMI had the least effect, it was eliminated from the model and re-analyzed to explore the possibility of body esteem having mediation effect. The simpler model with body esteem as the only mediator was significant $\left(\mathrm{R}^{2}=0.10, \mathrm{p}<0.05\right)$. The relationship between stigma consciousness and perceived physical activity competence was no longer significant $(\beta=-0.07, \mathrm{p}=0.71)$, but body esteem was $(\beta=0.28, \mathrm{p}=0.04)$ (fig. 3).

\section{Discussion}

Previous research has suggested that weight stigma negatively affects participation in healthy eating and coping behaviors, overall psychosocial well-being [5, 13, 14], and motivation for participation in physical activity [2, 4-6]. Although previous research explores how experience with or exposure to weightrelated stigma affects participation $[2,4,6]$, these studies have not taken into account the degree to which individuals are conscious of the stigma to which they have been exposed. In this study, stigma consciousness was measured to make the direct link between exposure to and awareness of stereotypes to consciousness. The results of this study indicate that consciousness of weight stigma affects perceived competence in physical activity, but that this relationship is mediated by body esteem. These findings are supported by the stigma consciousness construct which posits that individuals will curb their participation in an activity if they fear being stigmatized $[16,17]$. Furthermore, research exploring weight status perception suggests that individuals are subject to body esteem issues, and subsequently, weight-related stigma, regardless of their objective weight status [25]. The mediation of body esteem and lack of mediation of BMI adds a new dimension to previous research, suggesting that the subjective perception of weight (body esteem) can be more predictive of participation in physical activity than objective weight (BMI).

As posited, stigma consciousness of weight-related stigma was negatively related to perceived competence of physical activity, and body esteem was positively related to perceived competence of physical activity. BMI, however, was also related to perceived competence of physical activity, which was not predicted in the outset of the statistical analyses. Considering that BMI was not related to perceived competence of physical activity when the model was tested as a whole, suggests that there might have been some truth to the original hypothesis but that the relationship between BMI and perceived competence was confounded by the other two variables in the study.

The results in the present study are important in furthering professionals' understanding of the factors that affect behavior and subsequently conducting programs to overcome perceptions of weight and physical activity to encourage participation. The great amount of attention that has been paid obesity and the related health problems may be linked to an overall greater consciousness of weight, eating behaviors, body image, and self-perception. The results presented in this study suggest that obesity is as much a state of mind as it is an objective measure of weight. Consequently, individuals who have a poor body image or perceive themselves as being heavy are as susceptible to weight-related stigma as those who are not objectively overweight or obese. Current obsession with weight, and efforts to improve weight status, may negatively affect individuals who are already sensitive about their weight. Programmers should be cognizant of individuals' self-perceptions of weight, and their potential self-consciousness in participation in physical activity. Participants' stigma consciousness of weight, as much for people who have a healthy weight status as those who have an overweight status, may negatively affect their willingness to participate in physical activity. This might be especially the case if their motivation is to lose weight, if weight status is made salient during participation [32,33], or if there is a perception that participation in physical activity is for 'thin' people [7]. The providers' ability to recognize consciousness of weight-related stigma among participants, and differing degrees of stigma consciousness, would benefit the provider and the participant alike. Programs should incorporate a supportive environment in which participants are protected from the judgment of others. Counselors and providers should be trained in supportive methods of coaching, and to be sensitive to the detrimental effects of a negative self-perception. Furthermore, programmers and professionals who are part of the effort to improve weight-related issues in current society should be aware of how the movement is affecting the psyche of people of all weight statuses. More research toward this end is required to make recommendations, though it is possible that new angles and vocabulary should be introduced to change the public perspective on weight concerns.

\section{Limitations and Future Research}

The findings from this study are a valuable contribution to the direction research on obesity stigma should go; however, there are limitations that should be considered. First, the cross-sectional nature of the project limits the generalizability and predictability of direction and causality. A longitudinal project would provide more depth to the data, and offer opportunities to explore the effects of consciousness of weight stigma on perceived competence in physical activity over the long term. 
Additionally, this research incorporated individuals who were enrolled in a weight loss program, suggesting that they are motivated to change their weight status and social group membership perhaps more so than individuals who are not motivated to change their status. This project did not assess motivational factors to enroll in the program, of which perceived competence in physical activity might be one. Specifically, feeling as though they have the physical ability to make the necessary changes in their weight status may have encouraged these individuals to pursue a weight management program.

A comparison of individuals enrolled in the weight management program vs. those not enrolled may reveal an interesting contrast. The participants in this study are motivated to lose weight as they are already making efforts to change their weight status, be it subjective or objective. Yet, as indicated by the data presented in this study, they are still subject to weight stigma and their perceived competence in physical activity. Individuals who are not making efforts toward weight loss may have a different response to stigma consciousness and their perceived competence in physical activity. Research exploring how these variables exist differently for people of different motivations is needed in order to progress understanding. Another limitation to this study is that it was conduced with a mostly homogenous sample of white, non-Hispanic women. Future studies should incorporate a more diverse sample, or explore the effects of consciousness of weight stigma on perceived competence among women of other races and ethnicities, and on men. Consciousness of weight-related stigma, perception of weight and body esteem, and perceived competence in physical activity are likely to vary across ethnicities, races, and sexes.

Future research should incorporate an objective measure of physical activity (e.g., pedometer or accelerometer) to explore the relationships of social-psychological variables on actual participation. Although perceived competence is linked to participation, an objective measure of actual participation would be useful to better evaluate how stigma consciousness might affect participation and to what degree. Also, an objective measure of participation would be useful to evaluate the effectiveness of interventions designed to lessen stigma consciousness of weight-related stigma.

Another perspective would be to consider the attitudes an individual has towards others who are overweight or obese, and to assess individual motivations for participation in physical activity. The findings presented by this study that body esteem plays a role in an individual's perceived competence for physical activity warrant exploration into motivations for participation. Research has suggested that individuals who are motivated to participate in physical activity for weight loss have lower body esteem and subsequent perceived competence in physical activity than individuals who seek participation for its intrinsic rewards [2, 32-34].

The data presented in this study pose a viable argument that negative outcomes of weight stigma is not limited to people who qualify as being overweight or obese. Rather, individuals of all weight statuses are subject to potentially low levels of physical activity, psychological well-being, and subsequently physical health. As weight-related issues continue to garner attention and concern on an international level, individuals may become more aware and conscious of their own weight status, and allow themselves to become concerned, even when concern is not due (e.g., individuals of healthy weight status). Research exploring how weight-related issues and stigma affect people of all weight statuses will be useful in better serving physical and psychological health for the general public.

\section{Disclosure}

There are no conflicts of interests with the author and any sponsorship, funding agency, or data collection site.

\section{References}

1 Shaw SM, Henderson K: Gender analysis and leisure constraints: an uneasy alliance; in Jackson EL (ed): Constraints to Leisure. State College, PA, Venture, 2005.

2 Sykes H, McPhail D: Unbearable lessons: contesting fat phobia in physical education. Sociol Sport J 2008;25:66-96.

-3 Sallis JF, Hovell MF, Hofstetter CR: Predictors of adoption and maintenance of vigorous physical activity in men and women. Prev Med 1992;21:237251.

4 Rice C: Becoming 'The fat girl': acquisition of an unfit identity. Womens Stud Int Forum 2007;30: 158-174.

$\checkmark 5$ Storch EA, Milsom VA, DeBraganza N, Lewin AB, Geffken GR, Silverstein JH: Peer victimization, psychosocial adjustment, and physical activity in overweight and at-risk-for-overweight youth. J Pediatr Psychol 2007;32:80-89.
6 Vartanian LR, Shaprow JG: Effects of weight stigma on exercise motivation and behavior. J Health Psychol 2008;13:131-138.

7 Ball K, Crawford D, Owen N: Too fat to exercise? Obesity as a barrier to physical activity. Aust N Z J Public Health 2000;24:331-333.

8 Link BG, Phelan JC: Conceptualizing stigma. Annu Rev Sociol 2001;27:363-385.

9 Jones EE, Farina A, Hastorf AH, Markus H, Miller DT, Scott RA: Social Stigma: The Psychology of Sex Differences. New York, W.H. Freeman and Company, 1984.

10 Goffman E: Stigma: Notes on the Management of Spoiled Identity. Englewood Cliffs, NJ, Prentice Hall, 1963.

11 Centers for Disease Control and Prevention: Behavioral Risk Factor Surveillance System Survey Data. Atlanta, GA, Department of Health and Human Services, Centers for Disease Control and Prevention, 2007
12 Puhl RM, Brownell KD: Bias, discrimination, and obesity. Obes Res 2001;9:788-805.

13 Davison KK, Schmalz DL, Young LM, Birch LL: Overweight girls who internalize fat stereotypes report low psychosocial well-being. Obes 2008;16:S1S9.

14 Puhl RM, Moss-Racusin CA, Schwartz MB: Internalization of weight bias: implications for binge eating and emotional well-being. Obesity (Silver Spring) 2007;15:19-23.

15 Schwartz M, Vartanian L, Nosek B, Brownell KD: The influence of one's own body weight on implicit and explicit anti-fat bias. Obes Res 2006;144:440447.

16 Pinel E: Stigma consciousness: the psychological legacy of social stereotypes. J Pers Soc Psychol 1999;76:114-128.

17 Pinel E: Stigma consciousness in intergroup contexts. J Exp Soc Psychol 2002;38:178-185. 
18 Puhl RM, Latner JD: Stigma, obesity, and the health of the nation's children. Psychol Bull 2007; 133:557-580.

19 Friedman K, Reichmann S, Costanzo P, Zelli A, Ashmore J, Musante G: Weight stigmatization and ideological beliefs: relation to psychological functioning in obese adults. Obes Res 2005;13:907-916.

20 Crocker J, Major B: Social stigma and self-esteem the self-protective properties of stigma. Psych Rev 1989;96:608-630.

21 Davison K, Birch L: Predictors of fat stereotype among 9-year-old girls and their parents. Obes Res 2004;12:86-94.

22 Towler A, Schneider D: Distinctions among stigmatized groups. J Appl Soc Psychol 2005;35:1-14.

23 Quinn D, Crocker J: Vulnerability to the affective consequences of the stigma of overweight; in Swim J, Stangor C (eds): Prejudice: The Target's Perspective. San Diego, CA, Academic Press, 1998, pp. 125-143.
24 Wang SS, Brownell KD, Wadden TA: The influence of the stigma of obesity on overweight individuals. Int J Obes Relat Metab Disord 2004;28: 1333-1337.

25 Chang VW, Christakis NA: Self-perception of weight appropriateness in the United States. Am J Prev Med 2003;24:332-339.

26 Craft LL, Pfeiffer KA, Pivarnik JM: Predictors of physical competence in adolescent girls. J Youth Adolesc 2003;32:431-438.

27 Puhl RM, Brownell KD: Confronting and coping with weight stigma: a study of overweight and obese adults. Obesity 2006;14:1802-1815.

28 Harter S: Manual for the Self-Perception Profile for Children: Revision of the Perceived Self-Competence Scale for Children. Denver, CO, University of Denver, 1985.

29 Mendelson GK, Mendelson MJ, White DR: Body esteem scale for adolescents and adults. J Pers Assess 2001;76:90-106.
30 Chronbach LJ: Coefficient alpha and the internal structure of tests. Psychometrika 1951;16:297-334.

31 Baron RM, Kenny DA: The moderator-mediator variable distinction in social psychological research: conceptual, strategic, and statistical considerations. J Pers Soc Psychol 1986;51:1173-1182.

32 McCabe MP, Ricciardelli LA, Banfield S: Body image, strategies to change muscles and weight, and puberty: do they impact on positive and negative affect among adolescent boys and girls? Eat Beh 2001;2:129-149.

33 Galambos NL, Leadbeater BJ, Barker ET: Gender differences in and risk factors for depression in adolescence: a 4-year longitudinal study. Int J Beh Dev 2004;28:16-25.

34 Silberstein LR, Striegel-Moore RH, Timko C, Rodin J: Behavioral and psychological implications of body dissatisfaction: do men and women differ? Sex Roles 1988;19:219-232. 